\title{
Propuesta didáctica de organización de contenidos para la enseñanza de la genética en educación superior a distancia.
}

\author{
Luz Mery Bernal Parra ${ }^{1}$ \\ Gustavo Forero Acosta ${ }^{2}$ \\ Rene Montero Vargas ${ }^{3}$
}

\begin{abstract}
Resumen
A partir del reconocimiento de las situaciones que dificultan el aprendizaje de contenidos en el marco de la genética, se diseño una propuesta que maneja tres unidades conceptuales orientadas a viabilizar el proceso educativo de los estudiantes del Curso de Genética de la Universidad Nacional Abierta y a Distancia (UNAD) de Bogotá-Colombia. Es importante señalar que no se trata de una intención restringida al contexto de la educación abierta y a distancia, por el contrario, es un punto de partida para el fortalecimiento de los procesos educativos a partir de la reflexión sobre la propia práctica. En este sentido, se presentan las relaciones entre los contenidos de las tres unidades mencionadas y la estructura de competencias a evaluar en cada una de ellas, desde la idea de la interrelación de los contenidos y la construcción colectiva de saberes a partir de procesos de observación y análisis que integran la experiencia como un aspecto de suma importancia para la comprensión del estudiante.
\end{abstract}

Palabras clave: educación a distancia, enseñanza, enseñanza de las ciencias genética.

\footnotetext{
1. Doctora en Genética de la Universidad de Sao Paulo (Brasil). Docente Investigador de la Escuela de Ciencias Agrícolas Pecuarias y del Medio Ambiente de la Universidad Nacional Abierta y A Distancia UNAD Bogotá - Colombia. Correo electrónico: luz.bernal@unad.edu.co

2. Magister en Genética de la Pontificia Universidad Javeriana de Bogotá (Colombia).

Docente Investigador de la Escuela de Ciencias Agrícolas Pecuarias y del Medio Ambiente de la Universidad Nacional Abierta y A Distancia UNAD Bogotá - Colombia. Correo electrónico: gustavo.forero@unad.edu.co

3. Magister en Ciencias Biológicas de la Pontificia Universidad Javeriana de Bogotá (Colombia).

Docente Investigador de la Escuela de Ciencias Agrícolas Pecuarias y del Medio Ambiente de la Universidad Nacional Abierta y A Distancia UNAD Bogotá - Colombia. Correo electrónico: rene.montero@unad.edu.co

Está investigación está enmarcada dentro del proyecto "Aula Virtual para el desarrollo autónomo de competencias cognitivas: Integración de gestión de metas y contenidos mediante agentes inteligentes"
} 


\title{
A didactic proposal for content organization to teach genetics in distance higher education
}

\begin{abstract}
Based on the acknowledgement of situations hindering learning contents in the area of genetics, a proposal containing three thematic units aimed to facilitate learning processes for the students enrolled in the Genetics Course at Universidad Nacional Abierta y A Distancia (UNAD) is designed. It should be noted that this proposal is not restricted to open and distance learning, but a point of departure to strengthening educational processes from reflection on practice. In this sense, relationship between the contents in the three units aforementioned and the structure of skills to be evaluated in each is presented, based on the notion of content interrelation and collective knowledge construction from observation and analysis processes integrating experience as a key aspect for understanding among students.
\end{abstract}

Key words: distance learning genetics, teaching, science teaching.

Recibido: 21 de julio 2012

Aceptado : 22 de noviembre 2012

\section{Introducción}

Los aspectos relacionados con la enseñanza de la genética en la secundaria y los primeros niveles de los estudios de pregrado en carreras relacionadas con las ciencias, marcan una necesidad de avanzar en la comprensión de procesos y estrategias que den cuenta de posibilidades para la cualificación de la comprensión de los estudiantes en los temas que se tratan en la clases.

En este sentido, reconocemos que hay situaciones que pueden ser objeto de investigación, en cuanto al aprendizaje de la genética, dado que los estudiantes tienen algunas dificultades en el manejo de los contenidos (Cho, et al (1985) y Finley, et al (1982), los cuales son el punto de partida para pensar en retomar elementos que surjan de procesos de reflexión sobre la práctica pedagógica (Pérez, 
2005) y así, asumir retos en el marco de la enseñanza de las ciencias para sugerir alternativas que viabilicen nuestro trabajo.

Según Gagliardi (1986):

"Desde la síntesis de proteínas a la estructura de las poblaciones, desde la forma de expresarse los genes hasta el funcionamiento del sistema nervioso, hay muchos temas que no pueden aprenderse sin conocimientos previos de la biología y de otras ciencias.

¿Cómo comprender el funcionamiento celular sin un conocimiento básico en química?

¿Cómo llegar a definir la dinámica de poblaciones sin una base estadística? Todos los temas requieren un aprendizaje previo, todos son difíciles, todos son necesarios.

Es así que los programas de biología son generalmente muy largos, y que una mayoría de los alumnos sólo recuerda algunos nombres raros, pero no adquiere los conceptos que puedan permitirle continuar su aprendizaje si así lo desea, o, por lo menos, tener un panorama global del funcionamiento y las propiedades de los sistemas vivientes".

En este sentido la intención es generar una propuesta que permita integrar elementos conceptuales pedagógicos orientados a visibilizar en el estudiante procesos de construcción de conocimiento, de acuerdo con Corbacho (2009), el uso incorporara a las propuestas, actividades complementarias que permitan avanzar en el reconocimiento de situaciones en las que se emplean de una manera inadecuada los contenidos, es una posibilidad que debe tenerse en cuenta, dado que el hecho de contar con buenas respuestas frente a problemas, no es un sinónimo de comprensión por parte de los estudiantes, de lo cual es claro reconocer que para considerar la viabilidad de una propuesta pedagógica no solo deben tenerse en cuenta los resultados de las pruebas que se apliquen.

De acuerdo con lo anterior, se ha seleccionado el Curso de Genética de la Universidad Nacional Abierta y a Distancia (UNAD) de Colombia, para comenzar un proceso de reflexión en cuanto a la estructuración de contenidos, que permita que el estudiante reconozca la interdependencia entre ellos y le posibilite avanzar en la vía de la construcción de conocimiento como eje central de la formación.

En este sentido, es importante señalar que, para la propuesta que se presenta, se integran elementos desde la experiencia del estudiante (Bruner, 1973), la observación como un eje central del proceso educativo (Herrero, 1997), los análisis dentro del proceso argumentativo (Krippendorf, 1990) y las actividades 
diseñadas desde los problemas (Prieto 2006), de manera que se vinculan elementos suficientes para sugerir que el conocimiento sobre el contenido del curso de genética, se construye al desarrollar el curso.

\section{Referente Metodológico}

La propuesta generada hace parte de un estudio descriptivo en el marco de la reflexión sobre la propia práctica (Pérez, 2005) con base en el curso de Genética ofertado para estudiantes de los programas de pregrado de la Escuela de Ciencias Agrícolas Pecuarias y del Medio Ambiente (ECAPMA) de la Universidad Nacional Abierta y a Distancia (UNAD) de Colombia, en el marco del proyecto "Aula virtual para el desarrollo autónomo de competencias cognitivas: Integración de gestión de metas y contenidos mediante agentes inteligentes", desarrollado durante 2009 y 2010.

De la misma forma, se reconocen posiciones desde el trabajo de la interacción entre conceptos y las relaciones que dan lugar a la significación de los mismos (Patee, 1973), orientando hacia el manejo de redes conceptuales como una herramienta para el aprendizaje que también actúa desde la propuesta que se hace en cuanto a la enseñanza (Soussan, 2003).

También se reconoce como una posibilidad de trabajo en la propuesta, la visión de Gaulin (2001), frente a la resolución de problemas y sus posibilidades en el contexto de la enseñanza (Stewart, 1983; Martínez, 2006).

De acuerdo con lo anterior, se incluyen dentro del diseño de la propuesta y de los elementos para su evaluación, factores asociados con el uso de la experiencia en el contexto del aprendizaje de acuerdo con Bruner (1973) y la observación como generadora de posibilidades en dicho contexto (Herrero, 2007).

Con los elementos mencionados se construye la posibilidad de un diálogo entre el conocimiento del estudiante y el conocimiento científico, como posibilidad generadora en el marco del fortalecimiento de los procesos de enseñanza y aprendizaje en la educación abierta y a distancia, reconociendo que los problemas comunes que se encuentran actualmente en la formación de los estudiantes que ingresan a la educación superior, en lo que compete al conocimiento de lo genético, replican los mencionados por Cho, et al (1985) y Finley, et al (1982).

Señalamos que la propuesta se enmarca en el contexto de la educación a distancia, lo cual no es indicador de una dificultad para aplicarla en contextos presenciales, situación que generaría nuevas perspectivas para el conocimiento en torno a los procesos que se desarrollan en ambas modalidades y la pertinencia para el tratamiento conceptual en el contexto colombiano. 
En cuanto a los contenidos, la propuesta organiza la información de los temas empleando redes conceptuales como herramienta de organización de la enseñanza (Soussan, 2003), de manera que se cuenta con una estructura definida para comprender la intencionalidad de dichos contenidos, enfatizando en que no se requiere de una secuencia lógica para cada unidad, sino que cada contenido se interrelaciona con los demás desde posiciones que le permiten construir al estudiante una significación particular que depende de la manera como los articule (Patee, 1973).

Los contenidos que se manejan en la propuesta se articulan desde lo estructural y lo funcional como una herramienta orientada a la consolidación de la visión morfológica y fisiológica del evento genético.

El diseño de la propuesta se basó en dos fases: reconocimiento de criterios de evaluación en competencias y diseño de estructuras conceptuales para organización de contenidos.

\section{Fase1. Reconocimiento de Criterios de Evaluación en Competencias}

Para este aspecto se revisó la propuesta de competencias del curso de Genética de la UNAD y se consideraron los elementos descritos en dicho documento para definir la manera de articular los contenidos en la secuencia que brindara mejores elementos para la comprensión.

Se estableció un sistema de competencias para describir el estado de avance de los estudiantes con respecto al desarrollo de los contenidos, las cuales se relacionan con los procesos sugeridos en los documentos del curso. Para dicho trabajo se realizó la revisión de la propuesta de evaluación por competencias del Ministerio de Educación Nacional (MEN), de manera que se contó con un soporte desde las políticas educativas (MEN, 2006).

En este sentido las competencias sugeridas se enfocaron desde referentes conceptuales que se remiten a la posición desde lo pedagógico y lo didáctico relacionado con las ciencias.

Para el caso particular se definió trabajar una competencia conceptual siguiendo la posición de Coll (1987) quien menciona que "Independientemente del juicio que merezcan dichos conocimientos respecto de su pertinencia, adecuación, etc., lo que queremos señalar es su valor fundamental para el desarrollo de la persona que aprende, lo que justifica, a nuestro modo de ver, la importancia que adquieren los contenidos en la enseñanza", razón que sustenta el hecho de contar con espacios para reconocer el avance del estudiante en el dominio de los contenidos.

En cuanto al trabajo que se hace en la competencia contextual, reconocemos la importancia del contexto en la aplicación y significación de los contenidos, 
de manera que en palabras de Van Dijk (2001): "La teoría del contexto explica cómo los participantes son capaces de adaptar la producción y la recepción/ interpretación del discurso a la situación comunicativa interpersonal- social", y de acuerdo con eso, nuestra intención al plantear una competencia contextual, es precisamente dimensionar la posibilidad que tiene el estudiante de elaborar representaciones mentales que no son externas, visibles o se encuentran fuera de cada uno de ellos (Van Dijk, 2001).

A nivel de la competencia observacional, es importante mencionar que reconocemos la observación como un proceso fundamental en la construcción de la relación entre el estudiante y el conocimiento, en ese sentido consideramos que es necesario generar un espacio que permita valorar la manera en que el estudiante reconoce que "Observar es un proceso que requiere atención voluntaria e inteligencia, orientado por un objetivo terminal $u$ organizador, y dirigido a un objeto con el fin de obtener información", (De Ketele, 1984) y en ese sentido es capaz de aplicar procesos de observación en el contexto de la genética.

Al definir la competencia analítica, entendemos el aprendizaje como un proceso que requiere de elementos que lo constituyan y que se interrelacionen, de modo que el estudiante encuentre relaciones entre cada uno de los elementos que forman parte de su proceso educativo. En este sentido y de acuerdo con Krippendorff (1990), se entiende el análisis como "una técnica de investigación destinada a formular, a partir de ciertos datos, inferencias reproducibles y válidas que puedan aplicarse a su contexto"; nótese que desde el mismo planteamiento de las competencias, se procura establecer relaciones entre ellas, para conseguir un proceso evaluativo que se retroalimente desde sus mismos componentes.

En cuanto a lo interpretativo, se encontró que es necesario complementar la propuesta desde dicha posición dado que, como se mencionó anteriormente, es importante que los estudiantes encuentren conexiones en el proceso y en la evaluación del mismo, así que la interpretación como proceso dentro del trabajo de las ciencias experimentales, cobra importancia en atención a generar espacios donde el estudiante pueda interactuar con el contexto para reconocer situaciones que derivan del mismo (Soussan, 2003).

Por último, la competencia de aplicación obedece a la necesidad de involucrar el contexto (Van Dijk, 2001) y la experiencia (Bruner, 1973) en el proceso educativo. Es de mencionar que los demás procesos sugeridos en las competencias: análisis observación y conceptos, confluyen en ésta de modo que el estudiante construye explicaciones luego de poner en ejercicio los conceptos, inicialmente planos, que se presentaron.

De acuerdo con lo anterior, debe considerarse que la competencia de aplicación implica herramientas orientadas a generar posibilidades de acción en torno al uso 
de los contenidos; es así como partimos de reconocer que: "Dada la naturaleza del conocimiento científico en el aula de ciencias, deberían prevalecer interrogantes que supongan acciones como: describir ¿En qué consiste el hecho?, comparar ¿En qué radica la diferencia?, ¿Es posible encontrar semejanzas?, hipotetizar ¿Qué pensamos que va a ocurrir y por qué?, deducir Si....., entonces...¿Qué pasaría?, relacionar ¿Con qué se relaciona?, ¿Tiene que ver con algo que ya hemos visto? y explicar ¿Por qué crees que ocurre eso?, ¿Cómo explicamos que sea así?", (Leymonie, 2009); en este sentido involucramos posibilidades que permitan la integración de trabajo a partir de problemas (Coronel, 2008) y la integración conceptual en el marco de la posibilidad de motivar la generación de actitudes hacia la ciencia, desde el aprendizaje por investigación (Gagliardi, 1986; Prieto 2006; Martínez 2006)), toda vez que se pretende motivar la construcción de significaciones en el aprendizaje del contenido de la genética (Coll, 1987; 1988).

\section{Fase 2. Diseño de Estructuras Conceptuales para Organización de Contenidos}

En este sentido hemos recurrido a vincular tres posiciones en torno al manejo de los contenidos, con fines de viabilizar el aprendizaje del estudiante. Partimos de reconocer la importancia de contenidos que estructuren el conocimiento del campo específico según la propuesta de Gagliardi (1986) y Coll (1987), bajo la cual hemos identificado aquellos que son fundamentales en el contexto de cada unidad sugerida. Posteriormente, hemos reconocido las relaciones entre cada uno de ellos, para el diseño de estructuras conceptuales, de acuerdo con la reflexión que hace Soussan (2003) frente al uso de dichas estructuras como parte de la organización de la enseñanza y sus posibilidades en el aprendizaje en el marco de la enseñanza de las ciencias experimentales.

De acuerdo con lo anterior, consideramos que las estructuras que se diseñan deben permitir que en todo momento se cuente con la posibilidad de generar herramientas desde lo didáctico, dirigidas a integrar la experiencia del estudiante (Bruner, 1973) en el proceso de aprendizaje, de manera que se permita que la práctica educativa pueda permearse con el desarrollo de situaciones problema de acuerdo con la propuesta de Coronel (2008).

Sin embargo, la intención principal de la propuesta es lograr que el estudiante construya significaciones a partir del conocimiento de la disciplina, reconociendo que "el alumno aprende un contenido cualquiera - un concepto, una explicación de un fenómeno físico o social, un procedimiento para resolver determinado tipo de problemas, una norma de comportamiento, un valor a respetar, etc.- cuando es capaz de atribuirle un significado", (Coll, 1988).

Atendiendo entonces a sugerir una organización de contenidos con fines específicos en la construcción de significaciones, partimos de reconocer las dificultades que se presentan en el contexto de la enseñanza de la genética 
desde los estudios de Gagliardi, (1986), Cho, et al (1985), Finley, et al (1982) y Manassero (2001), donde se hace un reconocimiento de las situaciones más comunes que son generadoras de problemas en el aprendizaje de la genética.

En relación con los contenidos, establecimos un diálogo entre la visión de la genética desde la bioquímica (Nelson, 2007) y aquella que se tiene desde la biología molecular (Luque, 2005), de manera que se logra integrar situaciones desde la visión estructural y funcional, lo cual sumado a la propuesta de educación a nivel nacional en cuanto a lo genético (MEN, 2006), da como resultado una articulación dirigida a resaltar la idea de no secuenciar los contenidos, sino relacionarlos, de manera que el estudiante pueda jugar con ellos y sus interacciones con el fin de construir conocimiento (Soussan, 2003).

\section{Resultados y discusión}

\section{Fase 1}

De acuerdo con el análisis realizado a la propuesta del módulo del curso, se identificaron cinco competencias que el estudiante debía desarrollar al final del mismo; es de señalar que dicha categorización obedece a un ejercicio arbitrario que responde a las necesidades propias de los contenidos analizados y sugeridos en la propuesta, lo cual no va en contravía de las propuestas anteriores hechas por el MEN (2006), ni desconoce la estructura general de la evaluación por competencias.

Con base en esto se obtuvieron seis categorías de competencia:

1. Competencia Conceptual. Se ha establecido para que el estudiante pueda dar cuenta de las entidades conceptuales ya sean conceptos o contenidos que se encuentran dentro de un determinado tema.

2. Competencia Contextual. Se refiere al reconocimiento que el estudiante hace del contexto en el cual se plantea un determinado concepto, es decir los eventos que denotan la aparición del concepto mismo y se relacionan con eventos históricos que dan lugar a dicha aparición.

3. Competencia Observacional. Se refiere a los procesos relacionados con la observación de eventos relativos al concepto que se esté tratando, es de señalar que la observación no se entiende como un proceso meramente físico, sino que se relaciona con el reconocimiento y la posibilidad de relacionar aquello que se observa, la intención es posicionar la observación como un espacio de reconocimiento de los fenómenos que permite la construcción de conocimiento cuando se integra con las demás categorías. 
4. Competencia Analítica. Se define como la posibilidad de reconocer en un sistema de conceptos aquellas partes que lo componen, de manera que sirve como punto de partida para ir de lo general a lo particular y de esta forma tener la posibilidad de identificar un concepto o un contenido por los demás que lo componen, o llegar a dichos componentes simplemente por reconocer el concepto mismo.

5. Competencia Interpretativa. Se refiere a todas aquellas aptitudes del estudiante para establecer relaciones con el contexto donde se plantean las situaciones o donde aparecen los conceptos, es decir, la posibilidad de extraer información que no se ha brindado de primera mano, solo con reconocer las partes de una situación determinada o del contexto donde se propone el uso de un concepto determinado.

6. Competencia de Aplicación. Se refiere a emplear aquello que se conoce en un contexto definido por el usuario del mismo. Sin embargo, es importante señalar que la aplicación se pueden dar en contextos también sugeridos, caso en el que la presente competencia se enfoca en examinar la posibilidad de emplear un determinado concepto o grupo de ellos, de acuerdo con lo que se requiera explicar o sugerir. En síntesis, la integración del concepto y el contexto en la manera que se requiera para responder a un determinado cuestionamiento o necesidad dando cuenta del contenido y su aplicación.

Es necesario señalar que las categorías que se generaron responden a la necesidad encontrada en la propuesta de seguimiento a los temas definidos. Sin embargo, durante el proceso se encontró que las categorías contextual y conceptual podrían llegar a estar relacionadas desde el punto de vista de reconocer el concepto como una entidad necesariamente relacionada con un contexto dado que se enmarca en el mismo; sin embargo de acuerdo con Van Dijk (2001): “...los modelos del contexto se construyen como la base mental de eventos sociales de interacción y de comunicación, y como la base de la producción y comprensión discursiva...", lo cual permite comprender la separación que se hace entre concepto y contexto para establecer dichas categorías como entidades que viabilizan la identificación de procesos separados, en la construcción del estudiante. Caso similar que se encontró entre las competencias observacional, analítica e interpretativa, dado que reconocemos que los procesos de análisis involucran procesos relacionados con la observación y la interpretación; sin embargo, la razón para separarlos radica en la intención de reconocer el desarrollo de dichos procesos de manera independiente para luego reconfirmar el estado de los mismos en un nivel de integración en la competencia de aplicación.

En esta vía de acuerdo con De Ketele (1984), partimos de reconocer que: “... la observación es un proceso que requiere de atención voluntaria e inteligente orientada por un objetivo final...", de manera que asumimos separarla del 
análisis y la interpretación para reconocer la posibilidad del estudiante frente a reconocer aquello que se plantea como un "objetivo final", al momento de asumir su papel activo en el desarrollo de las temáticas y los fines propuestos para las mismas.

Por último, se ha considerado importante contar con una competencia de aplicación, de manera que sea posible identificar aquello que el estudiante puede hacer con la información que tiene en una situación determinada. Es de resaltar que la propuesta que se presenta no pretende enmarcarse en el contexto del aprendizaje significativo (Ausubel, 1973), aunque se intente establecer relaciones entre los contenidos y los aprendizajes.

La apuesta entonces, en cuanto a la intención de la competencia de aplicación, radica en apuntar a que pueda evidenciarse que el estudiante ha sido capaz de comprender un contenido o concepto cualquiera ${ }^{4}$ cuando puede otorgarle un significado en un contexto (Coll, 1988).

De esta manera, partimos de la idea de reconocer, en la competencia de aplicación, el valor de la experiencia dentro del proceso educativo como generadora de conocimiento y como medio de relación entre el sujeto y el contexto, de acuerdo con Bruner (1973):

"Antiguas experiencias, que tendían a oponer el aprendizaje mediante el descubrimiento personal al aprendizaje por procedimientos didácticos, $\mathrm{o}$, simplemente, comparando los medios de instrucción, tales como la televisión y los manuales, estuvieron lejos de conseguir su objetivo en el sentido de que no valoraban nada más que los conocimientos transmitidos, menospreciando la habilidad que se desarrolla de este modo".

Fase 2

Dada la revisión del modulo del curso que contiene los elementos básicos a trabajarse en el campo de lo genético y se estructura con base en la propuesta nacional (MEN, 2006), se reconocen tres grandes grupos temáticos a desarrollar:

1. Condensación del ADN y ciclo celular

2. Ácidos nucléicos y Gen

3. Transmisión de la información genética

Lo anterior obedece a la reflexión sobre la práctica realizada en años anteriores, por parte del equipo docente del curso académico de Genética, teniendo en cuenta

4. Para el caso particular nos referiremos a aquellos que se trabajan en el curso de Genética

5. EL autor se refiere al uso de la experiencia 
la propuesta que se hace en cuanto a organización de los contenidos en la Biología celular y Molecular de Luque (2005), considerando también la visión integral que se tiene en conjunto con la bioquímica y sus aplicaciones en el contexto de lo celular, de acuerdo con el tratamiento que hace de los contenidos el texto de Principios de Bioquímica de Lehninger (Nelson, 2007).

Para esto se establecen estructuras conceptuales, empleando la herramienta CmapTools, en cada uno de los temas que actúan como base para la interacción con estudiantes de manera que se sugiere emplear estas tres como unidades temáticas para el desarrollo del curso.

Las estructuras conceptuales de cada tema son las siguientes:

Condensación del ADN y ciclo celular.

Como se muestra en la figura 1, se ha diseñado una estructura conceptual con base en los contenidos del libro de Luque (2005), sin que se trate del seguimiento textual de la propuesta de dicho texto.

Se ha establecido que los conceptos que se muestran sean una base para el estudio de aquellos que están al interior de los mismos, de manera que trabajar con cualquiera de ellos, requiere el estudio de los demás.

De acuerdo con lo anterior se señala que una de las opciones, dentro de lo didáctico, para abordar el desarrollo y la aplicación de los contenidos sea la resolución de problemas, reconociendo que no es la única manera, así que seguimos la posición de Gaulin (2001) en cuanto a que: “...La decisión de tomar tal estrategia en lugar de tal otra, la decisión de continuar la investigación con tal estrategia en lugar de cambiarla, o la decisión de parar el trabajo y de cambiar de ruta para resolver todo esto, es metacognitivo ${ }^{6}$. Es una parte de lo que se llama metacognición, contiene una parte que sirve para controlar, para supervisar el trabajo y también para evaluar...".

Ahora bien, es importante mencionar que la organización que se presenta, desde la perspectiva de la enseñanza, obedece en palabras de Soussan (2003) a comprender que: "De hecho, cada nueva noción sólo puede ser comprendida, apropiada, memorizada, en función de las relaciones que ésta tiene con numerosas otras nociones del mismo campo conceptual. Por lo tanto, el dominio de una noción requiere el de todo un conjunto y el aprendizaje debe permitir a cada alumno la elaboración y la estructuración de este conjunto". 
Propuesta didáctica de organización de contenidos para la enseñanza de la genética en educación superior a distancia.

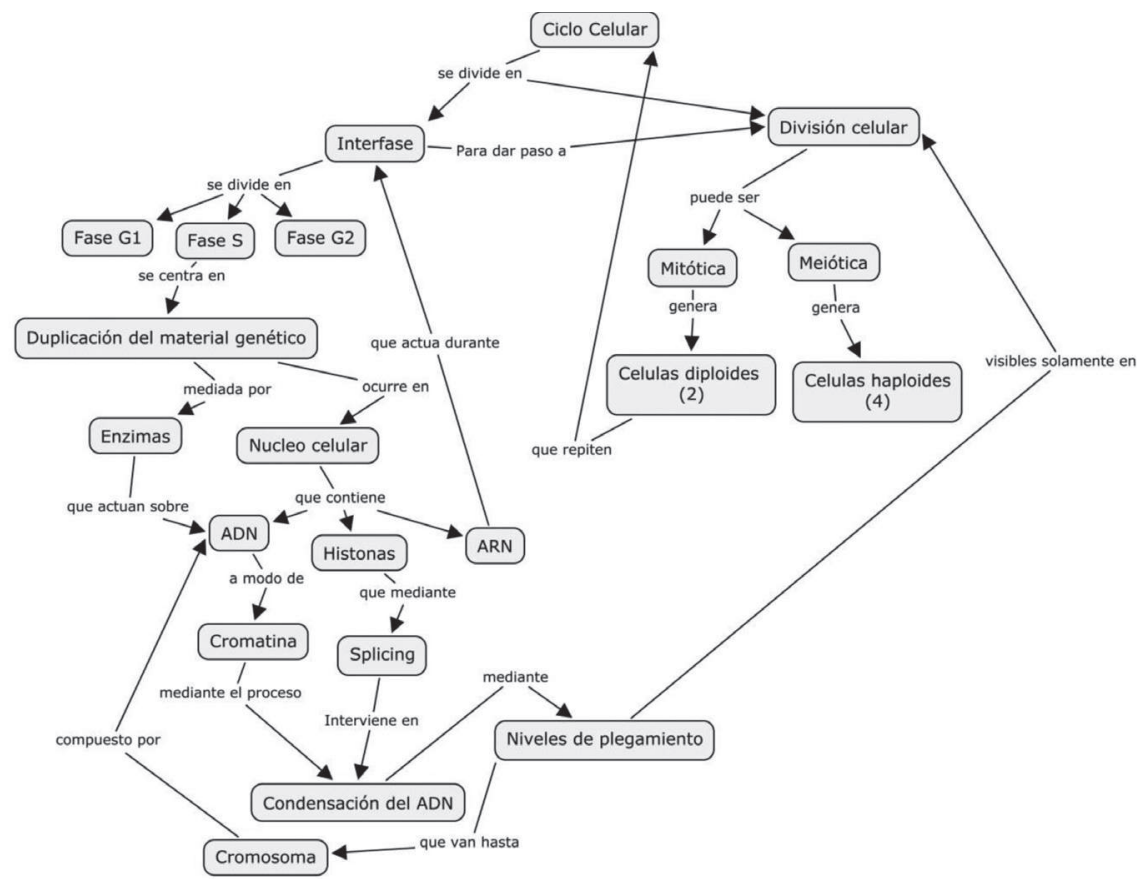

Figura 1. Mapa conceptual tema ciclo celular y duplicación del material genético.

Puede observarse la estructura de relación que se establece entre los conceptos involucrados en el tema mencionado, es de señalar que la organización obedece a la selección de contenidos que son base para tratar aquellos que no se mencionan, lo cual no indica que se vayan a eliminar de la propuesta. Se ha considerado que conceptos como ADN y ARN son fundamentales para navegar entre los temas propuestos de acuerdo con la propuesta de contenidos que maneja Luque (2005).

De acuerdo con lo anterior, sugerimos un esquema de competencias en la tabla 1, que pretende mostrar la posibilidad de integración que existe en torno a todos los elementos sugeridos en la construcción conceptual descrita anteriormente y que añade la intervención del estudiante directamente en su proceso formativo (Paredes, 2010), de modo que los contenidos no se muestren como entidades ajenas al estudiante y, por el contrario, se vean como estructuras esenciales al momento de iniciar el proceso del curso.

Se presenta la construcción de cada una de las competencias descritas en los resultados para la Fase 1 de acuerdo con el seguimiento conceptual que se hace del tema. Es de señalar la relación que se establece en cada una de las competencias entre el contenido conceptual y la acción del estudiante frente al proceso, así como la integración que se propone en la competencia de aplicación, la cual toma como base la propuesta de Paredes (2010), en torno a la importancia de la participación en el proceso educativo. 
Tabla 1. Competencias sugeridas para el tema Ciclo celular y Condensación del ADN.

\begin{tabular}{|c|c|}
\hline Tipo de Competencia & Competencia \\
\hline Conceptual & $\begin{array}{c}\text { El estudiante reconoce los conceptos histona, } \\
\text { cromatina, nucleosoma, solenoide, cromosoma y } \\
\text { ciclo celular en el marco de los mecanismos rela- } \\
\text { cionados con la condensación del ADN, mediante } \\
\text { el estudio de textos y situaciones que involucran } \\
\text { variantes en los mecanismos mencionados, con } \\
\text { el fin de identificar la integración y relación que } \\
\text { existe entre dichas estructuras conceptuales de } \\
\text { modo que se avance a la visión sistémica del } \\
\text { evento genético. }\end{array}$ \\
\hline Contextual & $\begin{array}{c}\text { El estudiante relaciona las entidades concep- } \\
\text { tuales relativas a la condensación del ADN y } \\
\text { ciclo celular con los escenarios donde ocurren, } \\
\text { indicando las variaciones o particularidades de } \\
\text { dichos procesos de acuerdo con las variantes } \\
\text { que los afectan de modo que consigue integrar el } \\
\text { concepto y el escenario desde la perspectiva de } \\
\text { la identificación de cada uno de ellos de manera } \\
\text { individual. }\end{array}$ \\
\hline Observacional & $\begin{array}{c}\text { El estudiante reconoce las estructuras que } \\
\text { aparecen durante el ciclo celular en láminas, es- } \\
\text { quemas, microfotografías o montajes y relaciona } \\
\text { directamente las características de cada uno de } \\
\text { los eventos de dicho proceso con las estructu- } \\
\text { ras observadas, de manera que reconoce los } \\
\text { procesos celulares involucrados en la condensa- } \\
\text { ción del ADN y el ciclo celular por las estructuras } \\
\text { propias de cada uno de ellos. }\end{array}$ \\
\hline Analítica & $\begin{array}{l}\text { El estudiante identifica el papel propio de cada } \\
\text { una de las estructuras que intervienen en el ciclo } \\
\text { celular y la condensación del ADN, argumentan- } \\
\text { do la razón de cada una de ellas en cada uno de } \\
\text { los procesos en que intervienen de manera que } \\
\text { consigue relacionar la particularidad del concepto } \\
\text { de cada estructura con la globalidad del evento } \\
\text { en que interviene. }\end{array}$ \\
\hline Interpretativa & $\begin{array}{l}\text { El estudiante reconoce en documentos científicos } \\
\text { los procesos y estructuras del ciclo celular y la } \\
\text { condensación del ADN, de acuerdo con los even- } \\
\text { tos presentados en los textos, de manera que } \\
\text { logre reconocer los procesos relativos a éstos } \\
\text { como consecuencia de los mismos aún cuando } \\
\text { no se traten explícitamente. }\end{array}$ \\
\hline De aplicación & $\begin{array}{l}\text { El estudiante integra el conocimiento de la fisio- } \\
\text { logía celular en el contexto del ciclo celular y la } \\
\text { condensación del ADN, señalando particularmen- } \\
\text { te las implicaciones de estructuras como histona, } \\
\text { cromatina, nucleosoma, solenoide y cromosoma, } \\
\text { en el marco de situaciones planteadas y varian- } \\
\text { tes de comportamientos fisiológicos celulares. }\end{array}$ \\
\hline
\end{tabular}




\section{Ácidos nucléicos y Gen}

Para este caso, se considera la propuesta de un manejo de contenidos integrando la visión que desde la bioquímica sigue el texto de Lehninger (Nelson, 2007) y desde la biología celular y molecular sigue el texto de Luque (2005). La idea central es reconocer la posibilidad de ver los contenidos desde diferentes ópticas, dada la dificultad que plantea Gagliardi (1986): "Hay numerosas investigaciones en las que se demuestra que las representaciones de los alumnos son diferentes a los conceptos que se les quiere enseñar".

De acuerdo con lo anterior, se ha pensado en articular los contenidos de manera que tengan sentido y haya su relacion entre ellos Figura 2, para que se consiga establecer puntos de comparación o ejemplos que permitan ver las implicaciones de dichos conceptos. En atención a esto, la estructura de contenidos se ha ideado con base en la propuesta de los sistemas jerárquicos de restricciones múltiples (Patee, 1973), que menciona que al establecer redes de conceptos se establecen entre ellos relaciones mutuas, que hacen que cada elemento tenga una significación única, lo cual genera que cada una de las significaciones que surja, lo hará como resultado de las interacciones que se establecieron entre los conceptos.

La estructura de red orientada a conseguir evitar el problema planteado por Gagliardi (1986), tiene su base en la propuesta que entiende las redes como herramientas diseñadas por el maestro para conseguir los objetivos que se traza en la enseñanza, así: "Las redes conceptuales son representaciones espaciales del campo científico al cual hace referencia una enseñanza determinada, en el ámbito de conocimientos y de estructuración deseable para el docente" (Soussan, 2003).

En cuanto al contenido de lo genético, hemos encontrado que esta forma de organización permite integrar los contenidos, de manera que el estudiante encontrará una amplia gama de posibilidades para abordarlos. La intención es mostrar que el conocimiento no tiene una secuencia específica siempre que se encuentre enmarcado en un campo delimitado por un fin específico, es decir, la manera de articular los contenidos obedece a la visión que se tenga del manejo de la disciplina en términos teleológicos.

Los elementos educativos que se hacen posibles al poner en acción esta propuesta dependen de la orientación que quiera darse con el componente del curso, considerando que esta organización obedece a una propuesta desde la enseñanza, bajo la cual el docente puede generar estrategias que permitan al estudiante construir procesos de aprendizaje.

En la misma vía es importante señalar que la estructura de mapas que se emplea, puede también usarse como estrategia para que el estudiante organice los contenidos que va trabajando, de manera que pueda establecer relaciones 
adicionales entre los contenidos, toda vez que dichas relaciones se construyen de acuerdo con la relación que se establezca entre el texto y el contexto.

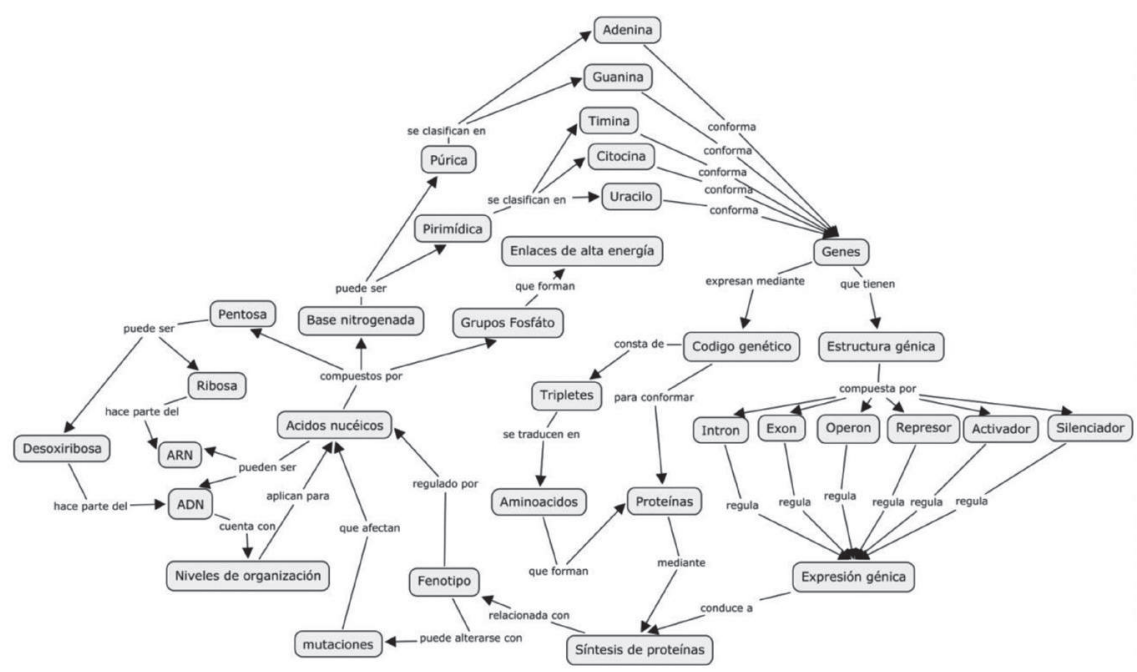

Figura 2. Mapa conceptual tema Ácidos Nucléicos y Gen.

Se muestra el énfasis que se hace en los contenidos desde la relación entre la composición bioquímica del ADN (Nelson, 2007), la estructura genética y la expresión del gen (Luque, 2005), de manera que se sugiere que sobre estos tres elementos se puede sustentar el trabajo de toda la unidad, sin desconocer el recorrido por los demás contenidos.

Vale la pena señalar que de acuerdo con la propuesta que se está haciendo, se sugiere un sistema de competencias, Tabla 2, ajustado a cada una de las situaciones particulares que se presentan en cada unidad. Partimos de reconocer que cada situación educativa es susceptible de sugerir una propuesta evaluativa dado que en palabras de Manassero (2001): "Más allá de detalles de estilo, metodología o preparación, la evaluación educativa tradicional ha consistido en comparar, para su valoración, las producciones del alumnado (escritas, orales, manuales, etc.), frente a un patrón de referencia establecido por el cuerpo de conocimientos disciplinar, que actúa como criterio último y universal", situación de evaluación que debe revisarse cuando el fin es que el estudiante construya conocimiento.

Se presenta la construcción de cada una de las competencias descritas en los resultados para la Fase 1 de acuerdo con el seguimiento conceptual que se hace del tema. Es de señalar la relación que se establece en cada una de las competencias entre el contenido conceptual y la acción del estudiante frente 
Propuesta didáctica de organización de contenidos para la enseñanza de la genética en educación superior a distancia. Artículo producto de la investigación.

al proceso, así como la integración que se propone en la competencia de aplicación, la cual toma como base la propuesta de Paredes (2010), en torno a la importancia de la participación en el proceso educativo.

Tabla 2. Competencias sugeridas para el tema ácidos nucléicos y Gen.

\begin{tabular}{|c|c|}
\hline Tipo de Competencia & Competencia \\
\hline Conceptual & $\begin{array}{l}\text { El estudiante reconoce los conceptos inherentes } \\
\text { a los ácidos nucléicos y el gen tales como pen- } \\
\text { tosas, bases nitrogenadas, nucleótidos, puentes } \\
\text { de hidrógeno, enlaces de alta energía, intrones, } \\
\text { exones y operones, en el marco de la dinámica } \\
\text { fisiológica nuclear, mediante el estudio de textos } \\
\text { que involucran la descripción de procesos pro- } \\
\text { pios de los ácidos nucléicos, con el fin de identifi- } \\
\text { car la estructura y el comportamiento bioquímico } \\
\text { que subyace a dichos conceptos. }\end{array}$ \\
\hline Contextual & $\begin{array}{c}\text { El estudiante relaciona los conceptos relativos a } \\
\text { los ácidos nucléicos celulares con el comporta- } \\
\text { miento fisiológico de los mismos en el contexto } \\
\text { de la fisiología genética-celular, para encontrar } \\
\text { explicaciones que den cuenta de las implicacio- } \\
\text { nes que tienen dichas moléculas en la expresión } \\
\text { génica. }\end{array}$ \\
\hline Observacional & $\begin{array}{l}\text { El estudiante reconoce la afinidad química entre } \\
\text { bases púricas y pirimídicas en esquemas, así } \\
\text { como la disposición espacial de las moléculas } \\
\text { de ácidos nucléicos relacionándolas con la es- } \\
\text { tructura del gen, de la misma manera identifica la } \\
\text { composición biológica de los genes y su relación } \\
\text { con la expresión de los mismos. }\end{array}$ \\
\hline Analítica & $\begin{array}{l}\text { El estudiante identifica la composición bioquímica } \\
\text { de las moléculas de los ácidos nucléicos como } \\
\text { parte esencial de la estructura génica donde pue- } \\
\text { de reconocer la relación morfológica y fisiológica } \\
\text { presente tanto en el comportamiento molecular } \\
\text { como en el biológico. }\end{array}$ \\
\hline Interpretativa & $\begin{array}{c}\text { El estudiante reconoce en documentos científicos } \\
\text { la función de los ácidos nucléicos y genes, de } \\
\text { acuerdo con los eventos presentados en los tex- } \\
\text { tos, de manera que logre reconocer la expresión } \\
\text { orgánica producto del comportamiento fisiológico } \\
\text { de los mismos. }\end{array}$ \\
\hline De aplicación & $\begin{array}{c}\text { El estudiante aborda situaciones que implican la } \\
\text { integración del conocimiento del comportamiento } \\
\text { bioquímico de los ácidos nucléicos y los genes, } \\
\text { las cuales requieren manejar información sobre } \\
\text { conceptos y procesos biológicos para sugerir } \\
\text { alternativas derivadas del conocimiento que se } \\
\text { tenga en cuanto a aspectos morfo fisiológicos de } \\
\text { la genética celular. }\end{array}$ \\
\hline
\end{tabular}




\section{Transmisión de la Información genética}

En este caso particular, hemos querido enfatizar en la necesidad de retomar el aspecto de la herencia desde la perspectiva molecular (Luque, 2005), siguiendo la intención de indicar que los conceptos que estructuran la disciplina, sugeridos en la Figura 3, hacen que el estudiante pueda seguir un curso de reconocimiento que permita llegar a construcciones conceptuales mediante las relaciones que se establecen entre los mismos (Gagliardi, 1986).

La propuesta de una unidad en este tema obedece al reconocimiento de las dificultades que presentan los estudiantes cuando salen de secundaria, dado que en esos niveles educativos es común encontrar que, en torno a la herencia y la genética se construyan explicaciones que difieren de las posiciones desde la ciencia, (Ayuso et al., 1996; Martínez et al, 2006).

Desde nuestra posición, pueden construirse explicaciones que den cuenta de los procesos científicos a partir del reconocimiento de situaciones particulares afines a la realidad de los estudiantes, así que consideramos que es importante articular a esta propuesta los elementos del aprendizaje basado en problemas (Prieto, 2006).

Es de señalar que el tratamiento sobre estos temas obedece también a que se conoce desde estudios anteriores que la enseñanza de la genética mendeliana, enfocada en la heredabilidad de los caracteres, genera problemas con el aprendizaje (Finley et al, 1982), de manera que los diagnósticos actuales sobre las dificultades en la enseñanza de la genética, recapitulan los hallazgos de dichos estudios.

De acuerdo con lo anterior, es importante mencionar que aún desde la década de 1980 existen preocupaciones por el manejo de este contenido, de modo que surgieron una serie de revisiones que dieron cuenta de las dificultades en cuanto al manejo de la terminología genética, la resolución de problemas en genética y las relaciones entre conceptos genéticos (Cho et al, 1982; Stewart, 1983; Radford et al 1985), específicamente en el marco del reconocimiento de la herencia como contenido de estudio genético.

Así las cosas, la organización de contenidos sugerida en la propuesta obedece a una estrategia para abordar situaciones particulares, que continúan ocupando la agenda del compromiso en la reflexión en torno a la enseñanza de la genética.

Por último, hay que señalar que los contenidos que se sugieren están en el contexto disciplinar genético molecular, sugerido por Luque (2005), el cual se funde con la idea de la construcción de conocimiento con base en la 
experiencia desde la idea de Bruner (1973) y el trabajo desde la resolución de problemas con el enfoque particular sugerido por Coronel et al (2008).

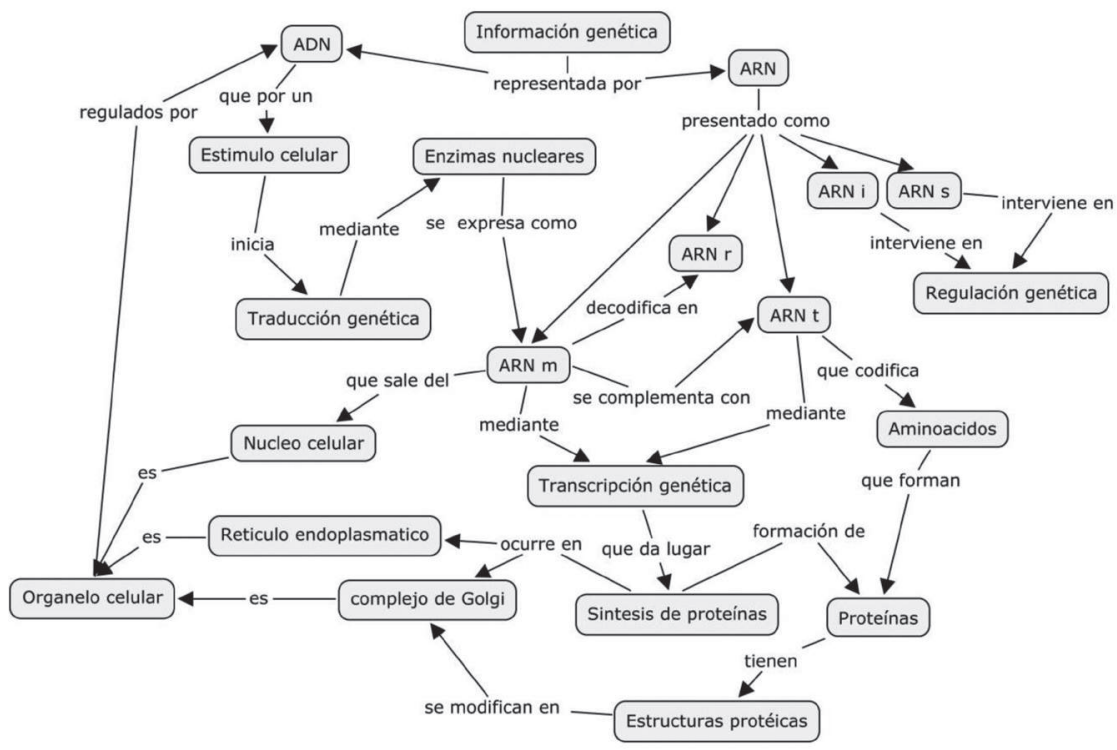

Figura 3. Mapa conceptual tema transmisión de la información genética.

Se muestra la propuesta de interrelación de los contenidos desde la idea de reconocer los elementos que generan confusión en la enseñanza de la genética (Martínez, 2006; Ayuso, 1996) e integrarlos de modo que den cuenta del contexto global del evento a tratar, desde la visión celular y molecular (Luque, 2005).

En cuanto a la propuesta de evaluación de esta unidad sugerida en la Tabla 3, debe considerarse el papel indiscutible que tiene el integrar la experiencia (Bruner, 1973) en el evento educativo para que pueda generar procesos que den lugar a la evaluación. En el mismo sentido, reconocer el papel que juega el trabajo a partir de problemas que integran la enseñanza (Ayuso, 199; Coronel, 2008), hace parte esencial de la propuesta, dado que se pretende integrar al estudiante en un proceso que le permita identificar situaciones que requieren de una integración conceptual para abordarse.

Se presenta la construcción de cada una de las competencias descritas en los resultados para la Fase 1 de acuerdo con el seguimiento conceptual que se hace del tema. Es de señalar la relación que se establece en cada una de las competencias entre el contenido conceptual y la acción del estudiante frente al proceso, así como la integración que se propone en la competencia de aplicación, la cual toma como base la propuesta de Paredes (2010) en torno a la importancia de la participación en el proceso educativo. 
Tabla 3. Competencias sugeridas para el tema transmisión de la información genética.

\begin{tabular}{|c|c|}
\hline Tipo de Competencia & Competencia \\
\hline Conceptual & $\begin{array}{l}\text { El estudiante reconoce las bases conceptuales } \\
\text { de los procesos de replicación, traducción, } \\
\text { transcripción del ADN y síntesis de proteínas, así } \\
\text { como sus implicaciones en los procesos celula- } \\
\text { res en que intervienen, mediante el estudio de } \\
\text { textos que involucran la expresión y regulación } \\
\text { génica a fin de identificar las relaciones existen- } \\
\text { tes entre código genético, proteínas y fenotipos. }\end{array}$ \\
\hline Contextual & $\begin{array}{c}\text { El estudiante reconoce los eventos relacionados } \\
\text { con la expresión y regulación de genes y síntesis } \\
\text { de proteínas, para ubicarlos en el contexto } \\
\text { celular para luego enmarcarlos en la dinámica } \\
\text { fenotípica, de manera que relaciona la expresión } \\
\text { de una entidad biológica con su funcionamiento } \\
\text { químico. }\end{array}$ \\
\hline Observacional & $\begin{array}{c}\text { El estudiante describe los procesos anexos a la } \\
\text { expresión de genes y la síntesis de proteínas de } \\
\text { manera que reconoce en esquemas y construc- } \\
\text { ciones conceptuales las vías implicadas en la } \\
\text { conservación del contenido genético de un orga- } \\
\text { nismo y aquellas que pueden generar variantes } \\
\text { en el mismo con las respectivas implicaciones en } \\
\text { la síntesis de proteínas. }\end{array}$ \\
\hline Analítica & $\begin{array}{c}\text { El estudiante reconoce la proteína como conse- } \\
\text { cuencia de la interpretación celular de un código } \\
\text { bioquímico que obedece a comportamientos } \\
\text { fisiológicos nucleares y se regula mediante } \\
\text { estructuras biológicas, señalando los procesos } \\
\text { que se requieren para conseguir la expresión de } \\
\text { los ácidos nucléicos en el contexto de la síntesis } \\
\text { de proteínas. }\end{array}$ \\
\hline Interpretativa & $\begin{array}{l}\text { El estudiante reconoce en documentos científicos } \\
\text { los mecanismos para regulación y expresión de } \\
\text { genes y síntesis de proteínas, de acuerdo con los } \\
\text { eventos presentados en los textos, de manera } \\
\text { que logre reconocer la relación existente entre } \\
\text { ácidos nucléicos y aminoácidos como base para } \\
\text { la conformación de fenotipos. }\end{array}$ \\
\hline De aplicación & $\begin{array}{l}\text { El estudiante ubica en el contexto de situaciones } \\
\text { definidas los elementos conceptuales, fisiológicos } \\
\text { celulares y fenotípicos implicados en la integra- } \\
\text { ción del comportamiento químico y biológico en } \\
\text { el marco de la función de genes y proteínas, de } \\
\text { manera que demuestra manejo de los contenidos } \\
\text { y aplicaciones de la genética en el contexto de } \\
\text { expresión y regulación génica y fenotípica. }\end{array}$ \\
\hline
\end{tabular}




\section{Conclusiones y Perspectivas}

El reconocimiento de las posibilidades educativas, que aparecen en la interacción y la reflexión sobre los resultados de las prácticas educativas propias, permite la generación de iniciativas enfocadas al mejoramiento de los procesos educativos nacionales con miras a la consolidación de prácticas exitosas en el marco de las necesidades particulares, más que en el de la modelización educativa.

Los conceptos y contenidos que aparecen en la reflexión de la práctica educativa pueden tener variaciones de acuerdo con la intencionalidad que se tenga en el evento educativo; sin embargo dicha variación aparece en la manera de trabajar con ellos y no en la presencia o ausencia de alguno, razón por la cual, en un ejercicio como el que se presenta debe cuidarse muy bien el aspecto disciplinar para conservar la estructura temática de cada uno de los contenidos que se trabajen, el eje debe ser el proceso pedagógico que subyace al tratamiento conceptual de la disciplina.

Es importante considerar que, como un ejercicio adicional a la presente propuesta, puede sugerirse iniciar espacios de reflexión frente a la pertinencia de emplear propuestas pedagógicas a modo de modelos puros en la práctica educativa, dado que señalamos que existen propuestas con aspectos valiosos que no necesariamente se centran en la reproducción literal de secuencias o instrucciones, por cuanto se trata de examinar estrategias educativas que se dirigen a estudiantes con diferencias en su relación con el conocimiento y en las posibilidades frente a la construcción del mismo.

La investigación educativa se consolida como un escenario adecuado para el reconocimiento de las fortalezas que se tienen en cuanto al proceso de enseñanza, de manera que deben buscarse alternativas para la consolidación de espacios y actitudes que se dirijan a posicionar la investigación y la reflexión sobre procesos educativos.

\section{Agradecimientos}

A la ECAPMA por su voluntad de crecimiento a partir del reconocimiento de sus propios procesos educativos.

\section{Referencias bibliográficas}

Ausubel, D. P. (1973). Algunos aspectos psicológicos de la estructura del conocimiento. En Elam, S. (Ed). Ed. El Ateneo. Buenos Aires. 
Ayuso, E., Banet, E., Abellán, T. (1996). Introducción a la Genética en la enseñanza secundaria y el bachillerato: resolución de problemas o realización de ejercicios. Enseñanza de las Ciencias, v.14 n², p.127-42.

Bruner, J., Olson, D. (1973). Aprendizaje por experiencia directa y por experiencia mediatizada. Revista Perspectivas-UNESCO. p.1-13.

Cho, H., Kahle, J., Nordland, F. (1985). An investigation of high school biology textbooks as sources of misconceptions and difficulties in genetics and some suggestions for teaching genetics, Science Education. v.69., $\mathrm{n}^{\circ} 5, \mathrm{p} .707-19$.

Coll, C., Solé, I. (1987). La importancia de los contenidos en la enseñanza. Investigación en la Escuela. V.3, p.19-28.

Coll, C. (1988). Significado y Sentido en el aprendizaje Escolar. Reflexiones en torno al concepto de aprendizaje significativo. Infancia y Aprendizaje. v.41., p. 131-42.

Corbacho, V. y De, P. (2009). Enseñanza de la genética en la educación de nivel superior: dificultades para comprender conceptos y resolver problemas. Enseñanza de las Ciencias, Número Extra VIII Congreso Internacional sobre Investigación en Didáctica de las Ciencias, Barcelona. p. 1021-24.

Coronel, M., Curotto, M. (2008). La Resolución de Problemas como estrategia de enseñanza y aprendizaje. Revista Electrónica de Enseñanza de las Ciencias. v.7., n²., p.463-79.

De Ketele, J.M. (1984). Observar para educar. $1^{\mathrm{a}}$. Ed. Madrid. Aprendizaje, Visor.

Finley, F., Stewart, J., Yarroch, W. (1982). Teacher's perceptions of important and difficult science content: The report of a survey. Science Education. v.66., p. 531-8.

Gagliardi, R. (1986). Los conceptos estructurales en el aprendizaje por investigación. Investigación y Experiencias Didácticas. v.4., n.1. p.30-5.

Gaulin, C. (2001). Tendencias actuales de la resolución de problemas. Conferencia En Sigma. v.19., p. 51-63.

Herrero, M. (1997). La importancia de la observación en el proceso educativo. Revista Electrónica Interuniversitaria de Formación del Profesorado. v.1., nº.o., p. 1-6.

Krippendorff, K. (1990). Metodología de análisis de contenido. Teoría y Práctica. Barcelona. Paidos.

Leymonié, J. (2009). Aportes para la enseñanza de las ciencias naturales. UNESCOLlece. Santiago de Chile 
Luque, J., Herraez, A. (2005). Biología Molecular e Ingeniería Genética. Elsevier. España.

Manassero, M. y Vásquez, Á. (2001). Instrumentos y métodos para la evaluación de actitudes relacionadas con la ciencia, la tecnología y la sociedad. Enseñanza de las Ciencias, v. 20., $\mathrm{n}^{\circ} 1$, p. 15-22.

Martínez, M., Ibañez, M (2006). Resolver situaciones problemáticas en genética para modificar las actitudes relacionadas con la ciencia. Enseñanza de las Ciencias, v. 24., $\mathrm{n}^{\circ} .2, \mathrm{p} .193-206$.

Ministerio De Educación Nacional-Men.(2006). Estándares Básicos de Competencias. $1^{\text {a }}$. ed. Bogotá, Imprenta Nacional de Colombia,.

Nelson, D (2007). Lehninger. Principios de Bioquímica. 5a Ed. Barcelona. Omega.

Paredes, E. (2010). Factores que afectan la participación de los estudiantes a distancia de la Universidad Rafael Belloso Chacín.

Redhecs. v.8., p. 63-81.

Patee, H.H. (1973). Hierarchy theory - The Challenge of Complex Systems. Nueva York. George Braziller.

Pérez. M. (2005). La investigación sobre la propia práctica como escenario de cambio escolar. Pedagogía Y Saberes. v. 18., p. $70-4$.

Prieto, L. (2006). Aprendizaje activo en el aula universitaria: el caso del aprendizaje basado en problemas. Revista de Ciencias Humanas y Sociales. v. 64., n¹24., p. 173-96.

Radford, A., Bird-Stewart, J. (1982). Teaching genetics in schools. Journal of Biological Education. v.6., n³. p. 177-80.

Soussan, G. (2003). Enseñar las Ciencias Experimentales, Didáctica y Formación. Ed Andros. Unesco - OREALC. Santiago de Chile.

Stewart, J. (1983). Student problem solving in high school genetics. Science Education, v. 67., p. 523-40.

Van Dijk, T. (2001). Algunos principios de una teoría del contexto. ALED. Revista latinoamericana de estudios del discurso. v. 1., nº., p. 69-81. 